\title{
Modelagem do efeito materno e estimativa de parâmetros genéticos para pesos corporais de caprinos Anglo Nubiano
}

\author{
Modeling the maternal effect and estimation of genetic parameters for body weights of \\ Anglo Nubian goats
}

SANTOS, Gleyson Vieira dos ${ }^{1 *}$; SOUSA, José Ernandes Rufino de ${ }^{2}$; SARMENTO; José Lindenberg Rocha ${ }^{3}$; SOUSA JÚNIOR, Severino Cavalcante de ${ }^{3}$; SOUSA, Wandrick Hauss de ${ }^{4}$; BIAGIOTTI, Daniel ${ }^{5}$; SANTOS, Natanael Pereira da Silva RÊGO NETO, Aurino de Araujo 5

\footnotetext{
${ }^{1}$ Universidade Federal do Piauí, Bom Jesus, Piauí, Brasil.

${ }^{2}$ Universidade Federal Rural do Semi-Árido, Departamento de Ciência Animal, Mossoró, Rio Grande do Norte, Brasil.

${ }^{3}$ Universidade Federal do Piaú, Departamento de Zootecnia, Bom Jesus, Piauí, Brasil.

${ }^{4}$ Empresa Estadual de Pesquisa Agropecuária da Paraíba, Setor de Produção Animal, João Pessoa, Paraíba, Brasil.

${ }^{5}$ Universidade Federal do Piauí, Teresina, Piauí, Brasil.

*Endereço para correspondência: gleysonvieira@zootecnista.com.br
}

\section{RESUMO}

Objetivou-se com este trabalho determinar o modelo mais adequado, bem como estimar os parâmetros genéticos do peso ao nascimento até os 196 dias de idade de caprinos da raça Anglonubiana. Os parâmetros genéticos foram estimados pelo método da máxima verossimilhança restrita (REML), utilizando o programa WOMBAT. Doze diferentes modelos animais foram ajustados por meio da inclusão ou exclusão do efeito genético materno, ambiente permanente materno, ambiente temporário materno e a covariância genética entre os efeitos direto e materno. Com base no critério de informação Bayesiano de Schwarz (BIC), o modelo II, que incluiu os efeitos genético direto e de ambiente temporário da mãe, foi o mais apropriado para todas as características. $\mathrm{O}$ efeito de ambiente temporário materno foi a mais importante fonte de variação. Este efeito contribuiu com $33 \%$ a $44 \%$ da variação fenotípica de acordo com o peso analisado. Estes resultados indicam que a seleção para efeitos direto e/ou materno em caprinos da raça Anglo-nubiana podem gerar progresso genético em características de crescimento.

Palavras-chave: ambiente permanente, componentes de variância, herdabilidade

\section{SUMMARY}

The aim of the study were to determine the most appropriate model, and to estimate genetic parameters of birth weight to the 196 day-old Anglo-Nubian breed goat. Genetic parameters were estimated by REML procedure using WOMBAT program. Twelve different animal models were fitted by including or excluding maternal genetic effect, maternal permanent environmental effect, maternal temporary environmental effect and covariance between direct-maternal genetic effects. On the basis of Bayesian information criterion results, model II, which included direct genetic and maternal temporary environmental effects, was determined to be the most appropriate model for both traits. The maternal temporary environmental effect was the most important source of variation for both traits. This effect contributed about $33 \%$ at $44 \%$ to the phenotypic variance for weight analized. These results indicate that selecting for improved maternal and/or direct effects in Anglo-nubian goats would generate genetic progress in growth traits.

Keywords: permanent environment, heritability, variance components 


\section{INTRODUÇÃO}

O melhoramento genético de caprinos de corte tem sido realizado em vários países e, mais recentemente, algumas tentativas têm sido conduzidas no Brasil para obter animais mais produtivos e eficientes. Medidas dos pesos corporais ao longo da vida do animal são informações importantes para avaliação genética de animais de corte, por ser uma alternativa barata e ao mesmo tempo de fácil mensuração.

A avaliação genética animal para características de crescimento depende das estimativas de parâmetros genéticos (herdabilidades e correlações genéticas), as quais podem ser influenciadas pelo modelo estatístico, pelo método de estimação dos componentes de (co)variância usados ou ainda pelos efeitos incluídos nos modelos. Portanto a procura por procedimentos, métodos e modelos que possam aumentar a acurácia das avaliações genéticas de animais constitui permanente desafio dos pesquisadores (SARMENTO et al. 2011).

As estimativas de parâmetros genéticos em caprinos, em sua grande maioria têm desconsiderado o efeito de ninhada (SARMENTO et al., 2003; BREDA et al., 2006; BOSSO et al., 2007; SOUSA et al., 2011a ; SOUSA et al., 2011b). A correta partição dos efeitos que podem influenciar no desempenho dos indivíduos, através da inclusão do componente genético aditivo direto e materno, bem como do ambiente permanente e temporário da mãe aqui denominado de efeito comum de ninhada, poderá fornecer estimativas de componente de covariância e herdabilidade não inflacionadas, resultando assim em predições mais acuradas dos valores genéticos. Neste sentido, a disponibilidade de modernos programas estatísticos e recursos computacionais têm facilitado o particionamento dos componentes de covariância, permitindo a análise de uma maior diversidade de modelos, fato que pode proporcionar melhores respostas à seleção.

Objetivou-se, portanto, determinar o modelo mais adequado, bem como estimar os parâmetros genéticos do peso ao nascimento até os 196 dias de idade de caprinos da raça Anglo-nubiana.

\section{MATERIAL E MÉTODOS}

Os dados utilizados foram registros de pesos corporais de borregos, filhos de 44 reprodutores e 279 matrizes de um rebanho experimental de caprinos da raça Anglo-Nubiana, provenientes do controle de desenvolvimento ponderal realizado em um rebanho pertencente à Empresa Estadual de Pesquisa Agropecuária da Paraíba (EMEPA-PB), com intervalos médios entre as pesagens de 28 dias. As características a serem estudadas são peso ao nascer (PN), peso aos 56 (P56), aos 112 (P112) e aos 196 (P196) dias de idade.

A propriedade onde foram coletados os dados situa-se no município de Soledade, nas coordenadas de $7^{\circ} 8^{\prime} 18^{\prime \prime} S$ e $36^{\circ} 27^{\prime} 2^{\prime \prime} \mathrm{W}$, com altitude média de $546 \mathrm{~m}$. Baseado na classificação de Köppen, o tipo climático da região é Bsh, semi-árido quente, apresentando temperaturas médias anuais em torno de $24{ }^{\circ} \mathrm{C}$, umidade relativa do ar em torno de $68 \%$, ocorrendo precipitação média de $400 \mathrm{~mm}$ anuais, com déficit hídrico durante quase todo o ano.

Os animais foram criados em sistema semi-intensivo em piquetes de pastagem nativa e nativa melhorada. $\mathrm{O}$ rebanho era submetido a períodos de monta controlada, com duração de 40 a 60 
dias, e início nos meses de março e novembro, com parições que se concentraram nos meses de fevereiro até abril e de agosto até setembro.

Os animais foram separados da mãe ao nascimento, receberam colostro três vezes ao dia, dieta sólida a partir do $10^{\circ}$ dia de vida e foram desaleitados no $70^{\circ}$ dia de idade, manejo adotado principalmente como forma preventiva da artrite-encefalite caprina (CAE).

Nas análises foram consideradas informações de reprodutores com no mínimo três filhos; cabras com no mínimo dois filhos e grupos contemporâneos com no mínimo três animais. Os efeitos fixos considerados na análise foram sexo da cria, tipo de nascimento e grupo de contemporâneos (formados pela combinação do ano com a estação de nascimento), além da covariável (linear e quadrática) idade da cabra ao parto.

Os componentes de covariância e os parâmetros genéticos foram estimados, utilizando o software WOMBAT (MEYER, 2006). Os modelos univariados foram ajustados para todas as características, considerando a covariância genética entre os efeitos direto e materno igual ou diferente de zero, como a seguir:

$$
\begin{array}{ll}
\text { Modelo } I: Y=X \beta+Z_{1} a+\varepsilon ; & \\
\text { Modelo } I I: Y=X \beta+Z_{1} a+Z_{3} t+\varepsilon ; & \operatorname{cov}(a, m)=0 \\
\text { Modelo } I I I: Y=X \beta+Z_{1} a+Z_{2} m+\varepsilon ; & \\
\text { Modelo } I V: Y=X \beta+Z_{1} a+Z_{4} c+\varepsilon ; & \operatorname{cov}(a, m)=0 \\
\text { Modelo } V: Y=X \beta+Z_{1} a+Z_{2} m+Z_{3} t+\varepsilon ; & \\
\text { Modelo } V I: Y=X \beta+Z_{1} a+Z_{3} t+Z_{4} c+\varepsilon ; & \operatorname{cov}(a, m)=0 \\
\text { Modelo } V I I: Y=X \beta+Z_{1} a+Z_{2} m+Z_{4} c+\varepsilon ; & \operatorname{cov}(a, m)=0 \\
\text { Modelo } V I I I: Y=X \beta+Z_{1} a+Z_{2} m+Z_{3} t+Z_{4} c+\varepsilon ; & \operatorname{cov}(a, m)=A \sigma_{a m} \\
\text { Modelo } I X: Y=X \beta+Z_{1} a+Z_{2} m+\varepsilon ; & \operatorname{cov}(a, m)=A \sigma_{a m} \\
\text { Modelo } X: Y=X \beta+Z_{1} a+Z_{2} m+Z_{3} t+\varepsilon ; & \operatorname{cov}(a, m)=A \sigma_{a m} \\
\text { Modelo } X I: Y=X \beta+Z_{1} a+Z_{2} m+Z_{4} c+\varepsilon ; & \operatorname{cov}(a, m)=A \sigma_{a m}
\end{array}
$$

em que $\mathrm{Y}=$ vetor $\mathrm{n} \times 1$ de observações; $\mathrm{X}=$ matriz de $\mathrm{n} \times \mathrm{f}$ de incidência, relacionado às observações aos efeitos fixos; $\beta=$ vetor $\mathrm{f} \times 1$ de efeitos fixos; $Z_{1}=$ matriz $\mathrm{n} \times \mathrm{N}$ de incidência, relacionado as observações aos efeitos 
genéticos aditivos diretos; $\mathrm{a}=$ vetor $\mathrm{N} \mathrm{x}$ 1 de efeitos genéticos aditivos diretos; $Z_{2}=$ matriz $\mathrm{n} \times \mathrm{N}$ de incidência relacionado às observações aos efeitos genéticos aditivos maternos; $\mathrm{m}=$ vetor $\mathrm{N} \times 1$ de efeitos genéticos aditivos maternos; $Z_{3}=$ matriz $\mathrm{n} \times \mathrm{P}$ de incidência relacionado às observações aos efeitos comum de ninhada, $\mathrm{t}=$ vetor $\mathrm{N}$ x 1 de efeitos comum de ninhada; $Z_{4}=$ matriz $\mathrm{n} \times \mathrm{M}$ de incidência, relacionado às observação dos efeitos de ambiente permanente materno, $\mathrm{c}=$ vetor $\mathrm{N} \times 1$ de efeitos de ambiente permanente materno; $\mathrm{A}=$ matriz de numeradores do coeficiente de parentesco entre os indivíduos, de ordem igual ao número de indivíduos (1.223); $\sigma_{\mathrm{am}}$ covariância entre os efeito genético direto e materno; $\mathrm{N}=$ número de indivíduos; $\mathrm{n}=$ numero total de observações; $\mathrm{f}=$ número de classes de efeitos fixos; $\mathrm{P}$ o numero de partos e $\mathrm{M}$ o numero de mães com observações.

A estrutura de variância e covariância para os efeitos aleatórios foram:

$$
\left\{\begin{array}{l}
V(a)=A \sigma_{a}^{2} \\
V(m)=A \sigma_{m}^{2} \\
V(c)=I_{d} \sigma_{c}^{2} \\
V(t)=I_{t} \sigma_{t}^{2} \\
V(e)=I_{n} \sigma_{e}^{2} \\
\operatorname{Cov}(a, m)=A \sigma_{a m}
\end{array}\right.
$$

em que A é a matriz de numeradores do coeficiente de parentesco entre os indivíduos, $\sigma_{a}^{2}$ a variância genética aditiva direta, $\sigma_{m}^{2}$ variância genética aditiva materna, $\sigma_{\mathrm{am}}$ covariância entre os efeito genético direto e materno, $\sigma_{c}{ }_{c}$ variância de ambiente permanente materno, $\sigma_{t}^{2}$ variância de ambiente temporário materno, $\sigma_{\mathrm{e}}^{2}$ variância residual, e $I_{d}, I_{t}, I_{n}$ são as matrizes identidade com ordem igual ao número de fêmeas, número de partos e número de observações, respectivamente.

Os diferentes modelos foram comparados utilizando os critérios de informação de Akaike (AIC) e bayesiano de Schwarz (BIC), que permitem comparações entre modelos não aninhados e impõem penalidades, de acordo com o número de parâmetros estimados. Para AIC e BIC, o valor para comparação é obtido conforme as equações:

$\mathrm{AIC}=-2 \log \mathrm{L}+2 \mathrm{pe}$

$B I C=-2 \log L+p \log (n-(r X))$,

em que: $\mathrm{p}$ é o número de parâmetros estimados; $\mathrm{n}$ é o número total de observações; e (r X) é o posto da matriz de incidência dos efeitos fixos no modelo. Menores valores para AIC e BIC indicam melhor ajuste. Além dos testes citados, os parâmetros estimados, para cada modelo, foram comparados para avaliar mudanças, em função do ajuste da variância residual.

\section{RESULTADOS E DISCUSSÃO}

As medias para as características estudadas (Tabela 1) foram 2,965; 9,$646 ; 13,887 ; 19,350 \mathrm{~kg}$ para peso ao nascer $(\mathrm{PN})$, peso aos 56 dias de idade (P56), peso aos 112 dias (P112), peso aos 196 dias (P196) de idade, respectivamente.

De acordo com os resultados apresentados em função dos critérios adotados (Tabela 2), ao considerarem os efeitos genético maternos, ambiente permanente e ambiente temporário da mãe observou-se melhoria significativa no ajuste dos demais modelos, quando comparado ao modelo com ajuste apenas do efeito genético aditivo direto (modelo I).

Ao analisar o modelo mais simples (modelo I), ou seja, aquele que 
Rev. Bras. Saúde Prod. Anim., Salvador, v.14, n.2, p.259-268 abr./jun., 2013 http://www.rbspa.ufba.br ISSN 15199940

considerou apenas o componente genético direto e o modelo, que incluiu o efeito de ambiente temporário da mãe (modelo II), observou-se maior redução nos valores de AIC e BIC para maioria das características, exceto para peso ao nascer e aos 196 dias de idade, sugerindo que houve uma melhoria no ajuste dos dados.

Tabela 1. Número de observações (n), média, desvio-padrão, coeficiente de variação (CV), mínimo e máximo para as características estudadas

\begin{tabular}{lcccccc}
\hline Característica & $\mathrm{n}$ & $\begin{array}{c}\text { Média } \\
(\mathrm{Kg})\end{array}$ & $\begin{array}{c}\text { Desvio- } \\
\text { padrão }(\mathrm{Kg})\end{array}$ & $\begin{array}{c}\mathrm{CV} \\
(\%)\end{array}$ & Mínimo & Máximo \\
\hline Peso ao nascer (PN) & 841 & 2,965 & 0,650 & 22,11 & 1,00 & 4,900 \\
Peso aos 56 dias de idade (P56) & 666 & 9,646 & 2,184 & 25,23 & 2,800 & 18,200 \\
Peso aos 112 dias de idade (P112) & 632 & 13,887 & 3,760 & 28,44 & 3,500 & 26,00 \\
Peso aos 196 dias de idade (P196) & 574 & 19,350 & 4,560 & 30,46 & 3,800 & 33,00 \\
\hline
\end{tabular}

Tabela 2. Critérios de Informação de Akaike (AIC) e Informação Bayesiano de Schwarz (BIC), para os modelos estudados

\begin{tabular}{ccccccccc}
\hline \multirow{2}{*}{ Modelos } & \multicolumn{2}{c}{ PN } & \multicolumn{2}{c}{ P56 } & \multicolumn{2}{c}{ P112 } & \multicolumn{2}{c}{ P196 } \\
\cline { 2 - 9 } & AIC & BIC & AIC & BIC & AIC & BIC & AIC & BIC \\
\hline I & $-40,82$ & $-31,44$ & 363,46 & 372,35 & 477,33 & 486,13 & 492,02 & 500,59 \\
II & $-56,45$ & $-42,39$ & 355,92 & 369,26 & 469,63 & 482,84 & 488,94 & 501,80 \\
III & $-46,53$ & $-32,46$ & 365,46 & 378,80 & 477,60 & 490,84 & 491,40 & 504,26 \\
IV & $-49,10$ & $-35,91$ & 365,47 & 378,80 & 477,77 & 490,97 & 490,85 & 503,71 \\
V & $-55,88$ & $-37,13$ & 357,93 & 375,72 & 471,49 & 489,10 & 490,45 & 507,60 \\
VI & $-57,13$ & $-38,38$ & 357,93 & 375,93 & 471,60 & 489,21 & 490,41 & 507,55 \\
VII & $-43,40$ & $-24,65$ & 367,47 & 385,25 & 479,41 & 497,02 & 492,52 & 509,67 \\
VIII & $-51,08$ & $-27,57$ & 359,93 & 382,16 & 473,50 & 495,50 & 492,27 & 513,70 \\
IX & $-44,81$ & $-26,06$ & 367,46 & 385,24 & 479,18 & 496,80 & 492,52 & 509,66 \\
X & $-54,04$ & $-30,60$ & 359,83 & 382,06 & 473,09 & 495,12 & 491,78 & 513,20 \\
XI & $-41,41$ & $-17,94$ & 369,46 & 391,69 & 480,83 & 502,85 & 493,72 & 515,15 \\
XII & $-49,05$ & $-20,89$ & 361,83 & 388,51 & 475,08 & 501,51 & 493,56 & 519,65 \\
\hline
\end{tabular}

Peso ao nascer (PN), peso aos 56 dias (P56), peso aos 112 dias (P112), peso aos 196 dias (P196) de idade.

Com base no AIC, o melhor modelo para característica peso ao nascer foi aquele que considerou o efeito genético aditivo direto, ambiente permanente e temporário da mãe (modelo VI), enquanto que de acordo com o valor de BIC o modelo que incluiu apenas os efeitos genético aditivo e ambiente temporário materno (modelo II) foi o que apresentou melhor ajuste dos dados. Uma possível explicação para essa divergência entre os critérios pode ser devido ao fato do BIC penalizar modelos com maior número de parâmetros que o AIC. 
Apenas para o peso ao nascer, o modelo que apresentou melhor ajuste conforme o critério de AIC foi o que considerou além dos efeitos aditivos diretos, os efeitos permanente materno e o comum de ninhada (modelo VI). Apesar da importância dos componentes genético e ambiental da mãe, observou-se que mesmo na fase inicial da vida, o efeito genético da mãe não apresentou grande influencia sobre o desempenho dos animais. De acordo com os valores de AIC e BIC, todos os modelos que inclúram o efeito genético materno, apresentaram os melhores resultados quando comparado com o modelo I, porém em nenhuma idade avaliada estes modelos proporcionaram melhor ajuste aos dados quando confrontados com o modelo II que considerou apenas $\mathrm{o}$ efeito de ninhada como efeito materno. Em estudo semelhante com ovinos, Hagger (1998) concluiu que em características de peso pré-desmame em pequenos ruminantes com partos múltiplos o efeito de ambiente temporários da mãe é mais importante que $o$ de ambiente permanente. Problemas temporário de saúde da ovelha, como pododermatite, mastite, ou desordem digestiva, afetam apenas os cordeiros de uma ninhada em particular, e não contribui para o efeito de ambiente permanente da ovelha.

A importância dos efeitos materno, para características de crescimento foi reportada por vários autores (MEYER, 1994; EKIZ, 2005; MANDAL et al., 2006), que observaram que sua contribuição do efeito diminuía após a desmama, porém esses efeitos ainda tinham pouca influencia nas idades seguintes, em particular o efeito de ambiente temporário.

Com base nos resultados apresentados, o modelo II, que considerou apenas o ambiente temporário da mãe como efeito materno foi o mais apropriado, indicando que este componente foi considerado o mais importante efeito materno para ambas as características analisadas.

A variância genética aditiva (Tabela 3.) para modelo I variou de $(0,10$ a $\left.2,15 \mathrm{~kg}^{2}\right)$ e foi consistentemente mais elevada do que as estimadas nos modelos II; V e VI $\left(0,02\right.$ a $\left.0,68 \mathrm{~kg}^{2}\right)$.

No modelo II, onde foram considerados os efeitos genético aditivo direto e de ambiente temporário da mãe, a variância genética aditiva sofreu uma redução de $50 \% ; 70 \%, 62 \%$ e $68 \%$ para os PN; P56, P112 e P196, respectivamente quando comparadas com as estimativas obtidas para $o$ modelo mais simples (modelo I). Além da variância aditiva direta, a variância residual também diminuiu consideravelmente em todas as idades, sugerindo que parte do efeito de ambiente temporário (ninhada) estaria sendo confundido com efeito residual no modelo I.

Com a inclusão do efeito genético direto materno (modelo V) o valor da variância atribuída ao efeito de ambiente temporário sofreu uma pequena redução, no entanto mantevese muito superior ao efeito genético materno por todo o período analisado. Comportamento semelhante foi observado para a variância residual, que foi ligeiramente superior para o modelo $\mathrm{V}$ do que no modelo II, evidenciando que os dados analisados apresentam-se melhor ajustados aos se considerar apenas os efeitos aditivo direto e de ambiente temporário da mãe.

O modelo que incluiu apenas o efeito temporário da mãe, como efeito materno foi considerado suficiente $\mathrm{e}$ mais indicado para o ajuste dos dados analisados, em relação ao efeito genético materno e permanente da mãe. 
Rev. Bras. Saúde Prod. Anim., Salvador, v.14, n.2, p.259-268 abr./jun., 2013 http://www.rbspa.ufba.br ISSN 15199940

Tabelas 3. Estimativas de componentes de covariância e parâmetros genéticos e fenotípicos, para as características estudadas

\begin{tabular}{|c|c|c|c|c|c|}
\hline Modelos & Parâmetros & $\mathrm{PN}$ & P56 & P112 & P196 \\
\hline \multirow{4}{*}{ I } & $\sigma_{{ }_{a}^{2}}^{2}$ & 0,10 & 0,70 & 1,90 & 2,15 \\
\hline & $\sigma_{e}^{2}$ & 0,18 & 2,44 & 5,70 & 9,53 \\
\hline & $\sigma_{p}^{2}$ & 0,28 & 3,14 & 7,60 & 11,68 \\
\hline & $\mathrm{h}_{\mathrm{a}}^{2}$ & 0,35 & 0,22 & 0,25 & 0,18 \\
\hline \multirow{6}{*}{ II } & $\sigma_{a}^{2}$ & 0,05 & 0,21 & 0,72 & 0,68 \\
\hline & $\sigma_{t}^{2}$ & 0,12 & 1,53 & 3,41 & 3,83 \\
\hline & $\sigma_{\mathrm{e}}^{2}$ & 0,10 & 1,47 & 3,49 & 7,08 \\
\hline & $\sigma_{p}^{2}$ & 0,27 & 3,21 & 7,62 & 11,59 \\
\hline & $h^{2}{ }_{a}$ & 0,19 & 0,06 & 0,09 & 0,06 \\
\hline & $t^{2}$ & 0,45 & 0,48 & 0,45 & 0,33 \\
\hline \multirow{8}{*}{ V } & $\sigma_{a}^{2}$ & 0,02 & 0,21 & 0,60 & 0,40 \\
\hline & $\sigma_{m}^{2}$ & 0,03 & 0,002 & 0,26 & 0,80 \\
\hline & $\sigma_{t}^{2}$ & 0,10 & 1,53 & 3,20 & 3,14 \\
\hline & $\sigma_{e}^{2}$ & 0,11 & 1,47 & 3,56 & 7,26 \\
\hline & $\sigma_{p}^{2}$ & 0,26 & 3,21 & 7,62 & 11,60 \\
\hline & $\mathrm{h}_{\mathrm{a}}^{2}$ & 0,09 & 0,06 & 0,08 & 0,03 \\
\hline & $\mathrm{h}_{\mathrm{m}}^{2}$ & 0,12 & 0,00 & 0,03 & 0,07 \\
\hline & $\mathrm{t}^{2}$ & 0,37 & 0,48 & 0,42 & 0,27 \\
\hline \multirow{8}{*}{ VI } & $\sigma_{a}^{2}$ & 0,02 & 0,20 & 0,60 & 0,25 \\
\hline & $\sigma_{t}^{2}$ & 0,09 & 1,53 & 3,28 & 2,90 \\
\hline & $\sigma_{c}^{2}$ & 0,04 & 0,001 & 0,18 & 1,09 \\
\hline & $\sigma_{e}^{2}$ & 0,11 & 1,48 & 3,55 & 7,31 \\
\hline & $\sigma_{p}^{2}$ & 0,26 & 3,21 & 7,61 & 11,55 \\
\hline & $\mathrm{h}_{\mathrm{a}}^{2}$ & 0,09 & 0,06 & 0,08 & 0,02 \\
\hline & $\mathrm{t}^{2}$ & 0,33 & 0,48 & 0,43 & 0,25 \\
\hline & $c^{2}$ & 0,16 & 0,00 & 0,02 & 0,09 \\
\hline
\end{tabular}

Peso ao nascer (PN), peso aos 56 dias (P56), peso aos 112 dias (P112), peso aos 196 dias (P196) de idade. $\sigma_{\mathrm{a}}^{2}=$ variância genética aditiva direta; $=\sigma_{\mathrm{m}}^{2}$ variância genética materna; $\sigma_{\mathrm{t}}^{2}$ variância de efeito ambiente temporário materno; $\sigma^{2}{ }_{c}$ variância de efeito ambiente permanente materno; $\sigma_{\mathrm{p}}^{2}$ variância fenotípica; $\sigma_{\mathrm{e}}^{2}$ variância residual. $\mathrm{h}_{\mathrm{a}}^{2}=$ herdabilidade genética aditiva direta, $\mathrm{h}_{\mathrm{m}}^{2}=$ herdabilidade genética aditiva materna, $\mathrm{t}^{2}=$ proporção da variância fenotípica devido variância de ambiente temporária materna $\mathrm{c}^{2}=$ proporção da variância fenotípica devido variância de ambiente permanente materna.

A redução na importância do efeito genético materno e de ambiente permanente da mãe pode ser atribuída ao manejo adotado no rebanho, uma vez que as crias eram separadas da mãe ao nascimento e mantidas sob aleitamento artificial até os 70 dias de idade, como forma preventiva da artrite-encefalite caprina (CAE).

Sarmento et al. (2006) em estudo com ovinos observaram que o efeito de ambiente permanente dificultaria a convergência no processo iterativo e valores confusos ou sem explicação biológica foram estimados, provavelmente em razão do pequeno volume de dados disponível e do baixo número de filhos por mãe.

As herdabilidades aditivas diretas obtidas pelo modelo I, foram superiores às estimadas pelos demais modelos, variando de 0,18 (para P196) a 0,35 (para $\mathrm{PN}$ ), conforme apresentado na Tabela 3. Semelhantemente ao que 
aconteceu com as variâncias genéticas aditivas diretas, as herdabilidades diretas estimadas por meio dos modelos II, V e VI foram consistentemente menores do que no modelo I (Tabela 3 ), dada a inclusão da variância atribuída ao efeito genético, ambiente permanente e temporário da mãe.

As estimativas de herdabilidade direta obtidas neste estudo estão dentro do intervalo reportado na literatura (MANDAL et al., 2006; BOSSO et al., 2007; SARMENTO et al., 2011) para diferentes métodos e raças.

No peso ao nascimento a herdabilidade direta diminui de 0,35 (modelo I) para 0,19 (modelo II) e depois para 0,09 (modelo V e VI). Para as demais idades observou-se uma diminuição da herdabilidade do modelo I para o modelo II, valores que permaneceram praticamente semelhantes entre 0 modelo II e os demais modelos (V e VI). Dessa forma, torna-se evidente que apenas para o peso ao nascimento, os efeitos genéticos da mãe e de ambiente permanente materno apresentaram maior influência (Tabela 2).

Apesar da importância destes efeitos, a contribuição do efeito de ambiente temporário da mãe foi muito mais expressiva, respondendo por 27 a $48 \%$ da variância fenotípica total, em função do modelo ajustado, sugerindo que o modelo que considerou o efeito genético aditivo direto e de ambiente temporário da mãe foi suficiente para ajustar os dados de caprinos analisados. As estimativas de herdabilidade para o efeito genético materno foram de magnitude baixa, variando de zero para P56 a 0,12 para PN. Verificou-se tendência de declínio do efeito materno logo após o nascimento (Tabela 3), divergindo da maioria dos resultados encontrados na literatura (SOUSA et al., 1999; QUESADA et al., 2002; SARMENTO et al., 2006).
Essa tendência já era esperada, devido ao manejo adotado onde os cabritos eram separados da mão ao nascer, porém fica evidente que o ambiente temporário de cada parto (ambiente temporário materno) teve grande influencia no desenvolvimento do animal, persistindo mesmo que com pouca intensidade, durante a fase adulta. Isso mostra que animais nascidos através de partos múltiplos têm grandes influencia desse ambiente comum, podendo ser influenciado até mesmo o ambiente uterino. Esses valores podem ser atribuídos ainda ao pequeno número de observações consideradas neste estudo, que possivelmente, dificultou a partição das variâncias entre os efeitos direto e materno pelo método empregado.

A proporção da variância fenotípica atribuída a variância de ambiente temporária da mãe foi de magnitude alta, variando de 0,25 a 0,48 . Com exceção do peso ao nascer, observou-se uma leve diminuição deste efeito com o aumento da idade, fato que pode ser atribuído a menor dependência dos animais aos efeitos maternos, à medida que os mesmos distanciam-se do nascimento. Isso indica que na avaliação genética e recomendados a inclusão do efeito de ambiente temporário materno.

A diminuição na magnitude do efeito de ambiente temporário da mãe com o aumento da idade pode ser atribuída também ao aumento das variâncias de efeito permanente da mãe. Outra possível explicação seria o grande número de partos compostos existentes no rebanho.

Torres Filho et al. (2004) trabalhando com suínos avaliando características de desempenho e reprodutivas concluiu que efeitos genéticos aditivo direto, materno e comum de leitegada devem ser considerados nos modelos. A 
importância do efeito de ambiente temporário materno em mamíferos também foi reportado por ( HAGGER, 1998; LEWIS \& BEATSON, 1999; Van WYK et al., 2003; Sousa et al., 2011b). $\mathrm{O}$ modelo II que considerou o efeito genético aditivo e de ambiente temporário da mãe foi o melhor modelo para estimação dos componentes de (co)variância e parâmetros genéticos para diferentes idades de pesagens em caprinos Anglo-Nubiano. Os valores de herdabilidade obtidas para peso nas idades consideradas indicam que é possível obtenção de progresso genético por meio de seleção.

\section{REFERÊNCIAS}

BOSSO, N.A; CISSÉ, M.F.; Van der WAAIJ, E.H.; FALL, A.; Van ARENDONK, J.A.M. Genetic and phenotypic parameters of body weigth in west African Dwarf goat and Djallonké sheep. Small Ruminant Research, v.67, p.271-278, 2007.

BREDA, F.C.; ALBUQUERQUE, L.G.; YAMAKI, M.; FILHO, J.C.R.; SARMENTO, J.L.R.; LOPES, P.S.; RODRIGUES, M.T. Estimação de parâmetros genéticos para produção de leite de cabras da raça Alpina. Revista Brasileira de Zootecnia, v.35, p.396404, 2006.

EKIZ, B. Estimates of maternal effects for pre- and post-Weaning daily gain in Turkish Merino lambs. Turkish Journal of Veterinary and Animal Sciences, v.29, p.399- 407, 2005.

HAGGER, C. Litter, permanent environmental, ram-flock, and genetic effects on early weight gain of lambs. Journal of Animal Science, v.76, p.452-457, 1998.
LEWIS, R.M.; BEATSON, P.R. Choosing maternal-effect models to estimate (co)variances for live and fleece weight in New Zealand Coopworth sheep. Livestock Production Science, v.58, p.137-150, 1999.

MANDAL, A.F.W.C.; ROUT, P.K.; ROY, R.; NOTTER, D.R. Estimation of direct and maternal (co)variance components for pré-weaning growth traits in Muzaffarnagari sheep.

Livestock Science, v. 99, p.79-89, 2006.

MEYER, K. Estimates of direct and maternal correlations among growth traits in Australian beef cattle.

Livestock Production Science, v.38, p.91-105, 1994.

MEYER, K. “WOMBAT” - Digging deep for quantitative genetic analyses by restricted maximum likelihood. In: WORLD CONGRESS OF GENETICS APPLIED TO LIVESTOCK PRODUCTION, 8, 2006, Belo Horizonte. Proceedings... Belo Horizonte, 2006.

QUESADA, M.; MCMANUS, C.; COUTO, F.A.D'ARAÚJO. Efeitos genéticos e fenotípicos sobre características de produção e reprodução de ovinos deslanados no Distrito Federal. Revista Brasileira de Zootecnia, v.31, p.342-349, 2002.

SARMENTO, J.L.R; PIMENTA FILHO, E.C.; RIBEIRO, M.N.; ARAÚJO, C.V. BREDA, F.C. PIRES, A.V.; TORRES FILHO, R.A.; TORRES, R.A. Fatores genéticos e de ambiente sobre o intervalo de partos de cabras leiteiras no semi-árido nordestino. Revista Brasileira de Zootecnia, v. 32, p.875-879, 2003. 
SARMENTO, J.L.R.; TORRES, R.A.; SOUSA, W.H.; PEREIRA, C.S.;

LOPES, P.S.; BREDA, F.C. Estimação de parâmetros genéticos para características de crescimento de ovinos Santa Inês utilizando modelos uni e multicaracterísticas. Arquivo

Brasileiro de Medicina Veterinaria e Zootecnia, v.58, n.4, p.581-589, 2006.

SARMENTO, J.L.R.; TORRES, R.A.; SOUSA, W.H.; LÔBO, R.N.B.; SOUSA, J.E.R.; CAVALCANTE NETO, A.; Ó, A.O. do. Efeito materno sobre a curva de crescimento de ovinos Santa Inês por meio de modelos de regressão aleatória. Comunicata Scientiae, v.2, p.113-121, 2011.

SOUSA, W.H.; PEREIRA, C.S.; BERGMANN, J.A.G.; SILVA, F.L.R. Estimativas de componentes de (co)variancia e herdabilidade direta e materna de pesos corporais em ovinos da raça Santa Inês. Revista Brasileira Zootecnia, v.28, p.1252-1262, 1999.

SOUSA, J.E.R; SILVA, M.A.; SARMENTO, J.L.R., SOUSA,W.H.; SOUZA, M.S.M.; FERREIRA, I.C. Estimates of covariance functions for growth of Anglo-Nubian goats. Revista Brasileira Zootecnia, v.40, p.106-114, 2011a.
SOUSA, J.E.R.; SARMENTO, J.L.R.; SOUSA, W.H.; SOUZA, M.S.M.; SOUSA JÚNIOR, S.C.; Ó, A.O.do; SANTOS, G.V. Aspectos genéticos da curva de crescimento de caprinos Anglo-Nubiano. Revista Brasileira de Saúde Produção Animal, v.12, p.340349, $2011 b$.

TORRES FILHO, R.A.; TORRES, R.A.; LOPES, P.S.; PEREIRA, C.S.; EUCLYDES, R.F.; ARAÚJO, C.V.; SILVA, M.A.; BREDA, F.C. Avaliação de Modelos para Estimação de Componentes de (co)Variância em Características de Desempenho e Reprodutivas em Suínos. Revista Brasileira Zootecnia, v.33, p.350-357, 2004.

VAN WYK, J.B.; FAIR, M.D.; CLOETE, S.W.P.; Revised models and genetic parameter estimates for production and reproduction traits in the Elsenburg Dormer sheep stud. South African Journal of Animal Science, v.33, p.213-222, 2003.

Data de recebimento: 29/01/2013

Data de aprovação: 20/05/2013 